\title{
Stefanie Freyer, Siegrid Westphal(dir.), Wissen und Strategien frühneuzeitlicher Diplomatie
}

\section{Roberto Berardinelli}

\section{OpenEdition}

\section{Journals}

Édition électronique

URL : http://journals.openedition.org/ifha/11047

DOI : 10.4000/ifha. 11047

ISSN : 2198-8943

Éditeur

IFRA - Institut franco-allemand (sciences historiques et sociales)

Référence électronique

Roberto Berardinelli, « Stefanie Freyer, Siegrid Westphal(dir.), Wissen und Strategien frühneuzeitlicher Diplomatie », Revue de l'IFHA [En ligne], Date de recension, mis en ligne le 28 décembre 2020, consulté le 30 décembre 2020. URL : http://journals.openedition.org/ifha/11047 ; DOI : https://doi.org/ 10.4000/ifha. 11047

Ce document a été généré automatiquement le 30 décembre 2020.

(C)IFHA 


\title{
Stefanie Freyer, Siegrid Westphal(dir.), Wissen und Strategien frühneuzeitlicher Diplomatie
}

\author{
Roberto Berardinelli
}

Ces dernières années, la recherche sur la diplomatie moderne a profité des nouvelles perspectives thématiques (voir les travaux de Lucien Bély, part. Lucien Bély, Espions et ambassadeurs au temps de Louis XIV, 1990) et des nouvelles approches théoriques de l'histoire culturelle (Barbara Stollberg-Rilinger (ed.), Was heißt Kulturgeschichte des Politischen?, 2005, p.9-26; Sven Externbrink, "Internationale Politik in der Frühen Neuzeit. Stand und Perspektiven der Forschung zu Diplomatie und Staatensystem ", dans: Christoph Kraus, Thomas Nicklas (dir.), Geschichte der

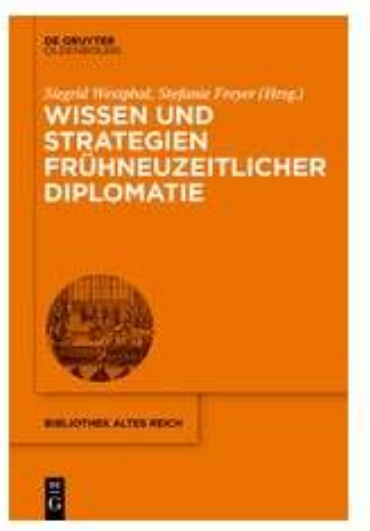
Politik, 2007, p.15-39). Une nouvelle historiographie de la diplomatie en a émergée dont le but n'est pas seulement la simple reconstruction des évènements politiques. Ils sont aussi analysés le modus operandi, les techniques et les stratégies des diplomates. La question décisive est comment les agents pratiquaient-ils la diplomatie à l'époque moderne, comment réussissaient-ils leurs missions?

Dans ce contexte ce n'est pas surestimer l'importance de l'ouvrage collectif Stefanie Freyer, Siegrid Westphal (dir.), Wissen und Strategien frühneuzeitlicher Diplomatie, Berlin/ Boston : De Gruyter Oldenbourg (Bibliothek Altes Reich, 27), 2020, 262 p., 59,95€ que de 
le qualifier d'une nouvelle référence dans l'histoire de la diplomatie moderne. Dans dix articles de 20-30 pages en moyenne les auteurs nous présentent des connaissances précieuses sur les pratiques diplomatiques au travers d'études de cas individuels biographiques. Entre autres nous faisons la connaissance des érudits, des envoyés, des nobles et voire même d'un musicien baroque. De cette manière l'ouvrage montre la diversité et la flexibilité étonnante des agents diplomatiques à l'époque moderne, une époque qui est caractérisée par le peu de professionnalisation et d'institutionnalisation dans les relations étrangères. Bien qu'il ne soit pas possible de donner une définition simple et précise du diplomate moderne nous pouvons constater que les auteurs essayent d'identifier et analyser les mécanismes essentiels de la diplomatie moderne et en faisant cela, l'ouvrage contribue à se rapprocher d'une sorte d'idéal-type weberien. Tous les articles ont en commun la perspective individuelle des acteurs mise en relation avec des réseaux sociaux. Ainsi, l'ouvrage suit l'approche méthodique des travaux de Hillard von Thiessen (Diplomatie und Patronage. Die spanisch-römischen Beziehungen 16051621 in akteurszentrierter Perspektive, 2010 ; - Akteure der Außenbeziehungen. Netzwerke und Interkulturalität im historischen Wandel, 2010).

Les contributions des différents auteurs sont précédées par une introduction de l'éditrice, Stefanie Freyer, qui propose un bilan historiographique (p. VII-XIII). Freyer y présente les faits typiques de la diplomatie moderne. Donc, l'introduction offre d'une orientation utile au lecteur, indifféremment aux experts et à tous ceux qui s'intéressent à l'histoire des relations internationales. Le livre est divisé en deux parties. La première partie traite la question fondamentale ce que les agents diplomatiques devaient savoir pour être capable de pratiquer la diplomatie. La deuxième section de l'ouvrage prend pour sujet les stratégies des diplomates en terme d'interaction à la cour et au dehors. La question plus importante est: de quelle manière les diplomates agissaient-ils pour réaliser leurs intérêts et leurs buts en mission. Autrement dit, l'ouvrage peut être considéré comme une analyse des conditions sine quibus non de la diplomatie à l'époque moderne: le savoir théorique-informatif et l'expérience pratique des agents diplomatiques comment communiquer et comment se comporter face aux élites courtoises.

Anna Lingnau propose une enquête historico-linguistique sur un rapport de l'envoyé brandebourgeois à la cour de Celle, Friedrich Rudolf von Canitz. En utilisant les méthodes de la linguistique politique Lingnau révèle le savoir politico-historique, linguistique et juridique qui était indispensable pour être capable de servir comme diplomate dans la deuxième moitié du XVIIe siècle (p.10-18). Au travers du traité «Getreuer Hofmeister auf Academien und Reisen » du savant Ehrenfried Walter von Tschirnhaus l'auteur Winfried Siebers recommande l'Apodemik (en français : l'art de voyager) comme un genre de sources propre pour éclairer les conditions comment les agents diplomatiques obtinrent d'une éducation convenable (p. 32-37). Dans son article Julian zur Lage s'intéresse à la genèse de l'ouvrage "History of America ", publié en 1777 par l'historien écossais William Robertson. Zur Lage montre que la référence à la collaboration de Robertson avec des envoyés anglais à Madrid et à Vienne augmenta le prestige et la légitimité du livre (p. 58-59). Les deux travaux suivants de la part de David Gehring et de Katharina Möhle thématisent la carrière diplomatique et les expériences confessionnelles de Robert Beale, un agent diplomatique et expert des relations anglo-impériales pendant le règne d'Élisabeth Ire. En analysant le rapport de Beale sur les affaires du Saint-Empire (The State of Germany) Möhle explique de quelle manière le diplomate diminue systématiquement la réputation du prince-électeur 
Auguste de Saxe en faveur de Frédéric III, électeur du Palatinat. Ce dernier est célébré comme un prince idéalement-chrétien qui, selon Beale, serait qualifié à une alliance protestante avec l'Angleterre élisabéthaine (p.91-100). L'article de Möhle souligne l'importance de la réputation princière et également l'importance du fait de la compromettre dans les relations étrangères à l'époque moderne.

La deuxième partie de l'ouvrage est introduite par la directrice elle-même. Stefanie Freyer analyse la stratégie de l'envoyé anglais à la cour de Vienne, Stephen Lesieur, consistant à mentir à l'Empereur Matthias Ier au sujet d'une alliance potentielle entre l'Angleterre et la monarchie française avec l'Union protestante au Saint Empire (p. 124130). Par conséquent, l'autrice livre une contribution estimable sur l'ampleur de la désinformation - qui, à notre avis, est encore sous-représentée dans la recherche internationale. Astrid Ackermann fournit une étude détaillée sur le réseau diplomatique européen de Bernhard de Saxe-Weimar, un military enterpriser pendant la Guerre de Trente Ans. Cette étude est intéressante parce qu'elle jette un éclairage sur les personnes militaires et semi-étatique qui initièrent aussi des missions diplomatiques (p. 140-142, 161-164). Volker Arnke conçoit un projet d'étude traitant un petit groupe d'envoyés inter-confessionnels allemands qui rendirent possible la conclusion du Paix de Westphalie (p. 168-171) et qui ont été longtemps négligés par l'historiographie (p.171-175). Recherchant à la jonction de la musique baroque et de l'histoire Claudia Kaufold reconstruit la carrière musico-diplomatique du compositeur italien Agostino Steffani aux cours de Munich, de Hanovre et de Bruxelles au tournant des XVIIe et XVIIIe siècles (part. p. 204-208). Steffani est un exemple impressionnant de la multifonctionnalité des agents diplomatiques à l'époque moderne. Pauline Puppel fait le portrait de la princesse Wilhelmine de Prusse qui chercha à provoquer une intervention prussienne aux Provinces-Unies en faveur de son mari, le stathouder Guillaume V d'Orange-Nassau, au zénith du conflit entre le mouvement patriotique et les défenseurs du stathoudérat en 1787 (p. 235-238, 241-242). L'article est un plaidoyer pour faire attention aux femmes comme agents et décideurs dans la diplomatie à l'époque moderne (p. 247-248).

Dans leur ensemble, les articles sont appuyés sur un solide appareil de sources. Lingnau, Möhle, Freyer, Kaufold et Puppel, en particulier, prouvent l'importance de ressusciter les vies des diplomates et négociateurs du second ou même troisième rang à partir les fonds d'archives et de le combiner avec des approches méthodiques actuelles comme font Lingnau avec la linguistique politique, Möhle et Freyer avec l'histoire des sensibilités, Gehring et Ackermann avec l'analyse des réseaux sociaux, Arnke avec les negotion studies et Puppel avec l'histoire du genre.

On pourrait critiquer que le titre "Wissen und Strategien frühneuzeitlicher Diplomatie " manque de la précision, il implique une perspective européenne, voire même globale. À l'exception des études d'Ackermann, de Kaufold et de Puppel le livre est un peu trop limité au Saint-Empire, à l'Angleterre et aux relations anglo-impériales. Cependant cela ne diminue pas le grand mérite du livre d'avoir capturé les mentalités et les pratiques de la diplomatie à l'époque moderne. Grâce aux études de cas les auteurs ont réussi à expliquer comment la flexibilité, la mobilité, les dépendances clientélistes et la multifonctionnalité sociale des agents diplomatiques caractérisent la diplomatie à l'époque moderne. Nous recommandons vivement ce livre à tous les experts des relations étrangères et également à tous ceux qui désirent le devenir. 
INDEX

Index chronologique : Période moderne

Thèmes : Histoire des États et des pouvoirs

\section{AUTEUR}

ROBERTO BERARDINELLI

Universität Heidelberg 